\title{
Evolución de la incidencia de migraña en Álava en el período 2004-2008
}

\author{
Luis Carlos Abecia-Inchaurregui, Natalia Burgos-Alonso, M. Eugenia Azpeitia-Serón, \\ Enrique Echevarría-Orella, Ismael Barbero-Martínez, Fernando Rodríguez-Artalejo
}

Introducción. Existen muy pocos estudios sobre la incidencia de migraña en España, y los que hay tienen alguna limitación, como carecer de base poblacional clara.

Objetivo. Examinar la evolución de la incidencia de migraña a lo largo del período 2004-2008 en la provincia de Álava (País Vasco, España).

Pacientes y métodos. Los datos corresponden a personas de 15 o más años de edad registrados como nuevos casos diagnosticados de migraña en la base de datos del Servicio Vasco de Salud/Osakidetza. La tendencia en la incidencia de migraña se valoró mediante regresión lineal.

Resultados. La incidencia de migraña fue significativamente mayor en las mujeres que en los hombres, a cualquier edad. No se observaron cambios en la incidencia de migraña en el período 2004-2008 en el conjunto de la población de Álava $(p=0,189)$. Sin embargo, se ha producido un descenso en la incidencia de migraña en las mujeres mayores de 64 años $(p=0,014)$, y un aumento en las de $15-24$ años $(p=0,052)$ y 35-44 años $(p=0,057)$. Los nuevos casos de migraña diagnosticada tendieron a aparecer en edades más jóvenes a lo largo de este período.

Conclusiones. Estos resultados indican una ausencia de cambios en la tendencia de la incidencia de migraña a lo largo del período 2004-2008, excepto un descenso en el caso de las mujeres mayores de 64 años y un aumento en las jóvenes. Esta evolución es similar a la de otras regiones europeas.

Palabras clave. Incidencia. Migraña.

\section{Introducción}

La migraña es una patología crónica caracterizada por dolores de cabeza recurrentes acompañados de náuseas, vómitos y sensibilidad a la luz y al sonido [1]. Es la patología neurológica más frecuente en Europa, con una prevalencia de alrededor del $12 \%$, y afecta dos veces más a las mujeres que a los hombres [2].

Se ha observado que la migraña -y especialmente la migraña con aura- es un factor de riesgo de enfermedad cerebrovascular, sobre todo en el sexo femenino, al consumir tabaco y usar contraceptivos clásicos [3]. Además, se ha demostrado recientemente que la migraña con aura es un factor de riesgo independiente para la mortalidad cardiovascular y de otros tipos, en hombres y mujeres [4].

Aunque muchos migrañosos nunca han consultado a un médico, la migraña es una causa frecuente de incapacidad temporal, con un alto impacto tanto clínico como social [5]. Desde esta perspectiva social, la migraña representa también un considerable coste económico en España, especialmente por la pérdida de productividad asociada [6].
La migraña es también uno de los trastornos neurológicos más comunes en España [7], con una prevalencia estimada del $16,6 \%$, según los datos de un cuestionario autoadministrado [8], y del 11,02\%, según los datos de la Encuesta Nacional de Salud de España del año 2006 [9]. En un reciente estudio realizado a través de entrevistas telefónicas a más de 70.000 personas, se ha estimado la prevalencia en el $12,6 \%$ [10]. Sin embargo, los estudios basados en población sobre la incidencia de la migraña en España son escasos.

Por ello, el objetivo del trabajo fue estimar la incidencia de casos diagnosticados médicamente de migraña en la región de Álava (País Vasco, España) y examinar su tendencia en el período 2004-2008.

\section{Pacientes y métodos}

El trabajo se realizó con datos procedentes de pacientes de la provincia de Álava, incluidos en el sistema informático del Servicio Vasco de Salud/Osakidetza. El período del estudio abarcó desde el 1 de
Departamento de Medicina Preventiva y Salud Pública (L.C. Abecia-Inchaurregui, N. Burgos-Alonso, M.E. AzpeitiaSerón); Departamento de Fisiología (E. Echevarría-Orella, I. BarberoMartínez); Facultad de Farmacia; Universidad del País Vasco; Vitoria, Álava. Departamento de Medicina Preventiva y Salud Pública; Facultad de Medicina; Universidad Autónoma de Madrid-CIBERESP (F. RodríguezArtalejo); Madrid, España.

Correspondencia:

Dr. Luis Carlos Abecia Inchaurregui. Departamento de Medicina Preventiva y Salud Pública. Facultad de Farmacia. UPV/EHU. P.․ Universidad, 7. E-01006 Vitoria-Gasteiz (Álava).

Fax:

+34945013014.

E-mail:

luiscarlos.abecia@ehu.es

Aceptado tras revisión externa: 08.02.12.

Cómo citar este artículo: Abecia-Inchaurregui LC, BurgosAlonso N, Azpeitia-Serón ME, Echevarría-Orella E, BarberoMartínez I, Rodríguez-Artalejo F. Evolución de la incidencia de migraña en Álava en el período 2004-2008. Rev Neurol 2012; 54: 537-43.

(c) 2012 Revista de Neurología 
Tabla I. Tasa de incidencia (I) × 100.000 habitantes por sexo y grupos de edad (año 2004).

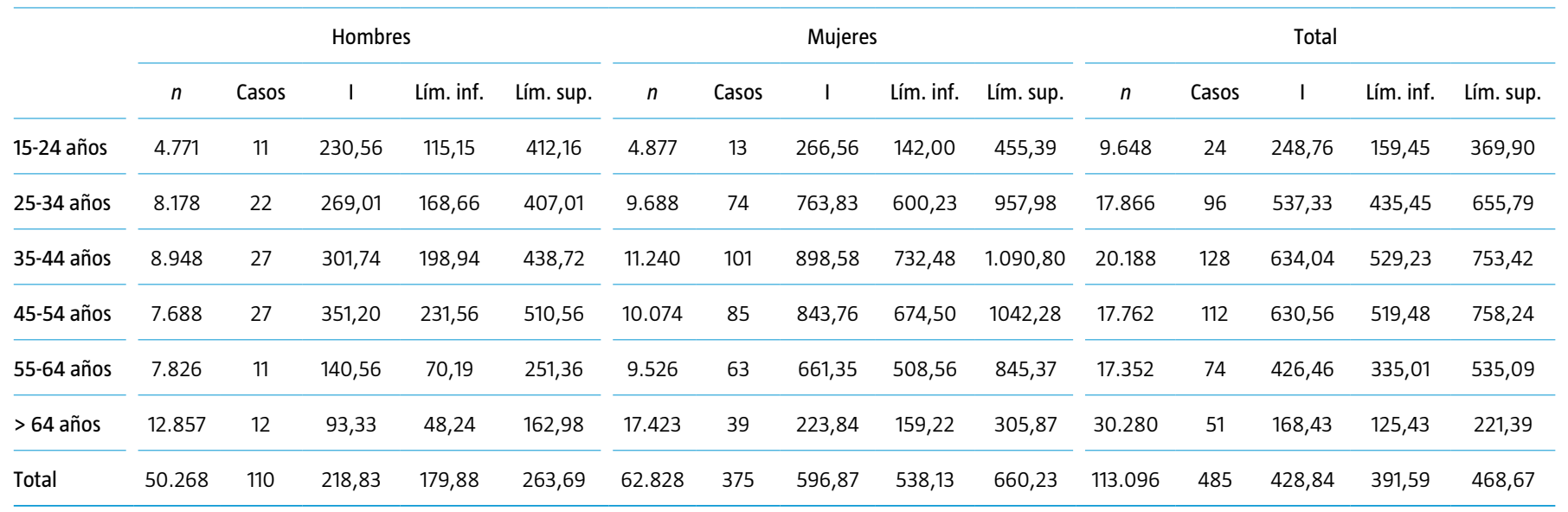

Tabla II. Tasa de incidencia (I) × 100.000 habitantes por sexo y grupos de edad (año 2005).

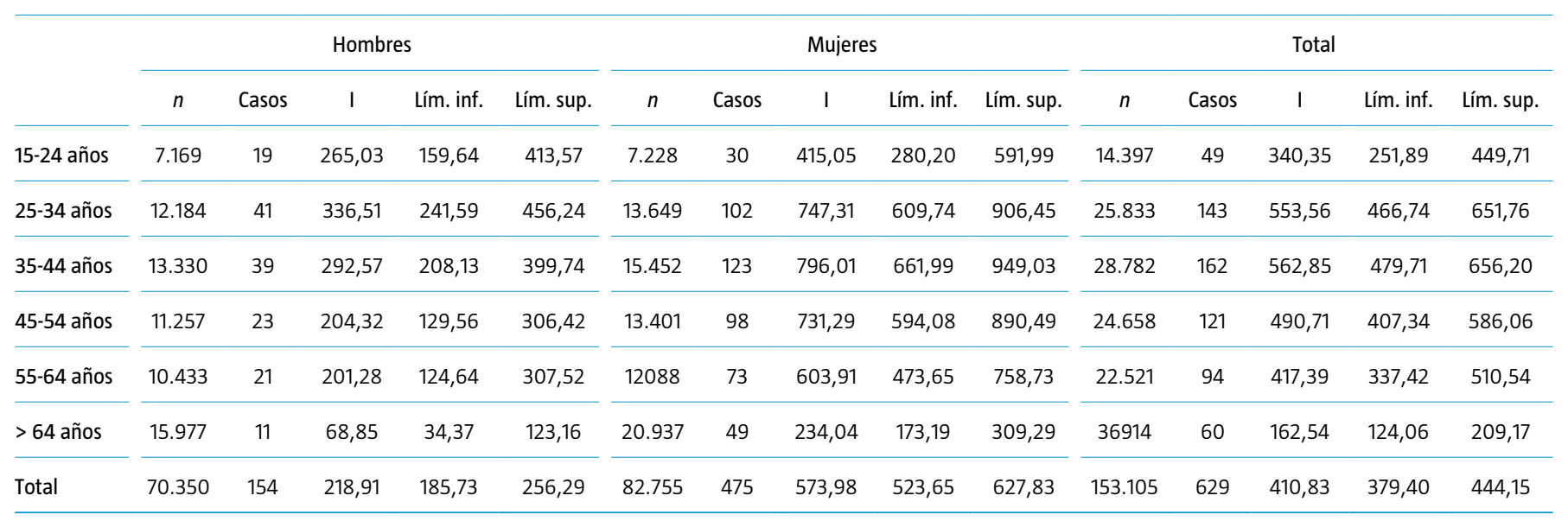

Tabla III. Tasa de incidencia (I) × 100.000 habitantes por sexo y grupos de edad (año 2006).

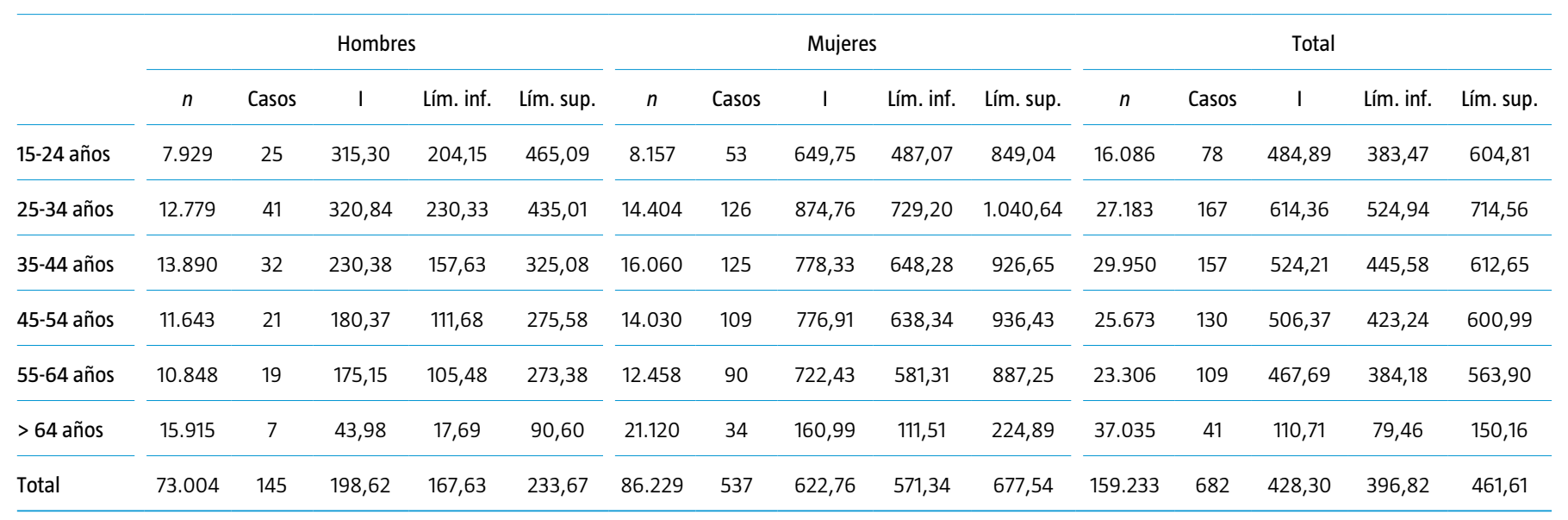


Tabla IV. Tasa de incidencia (I) $\times 100.000$ habitantes por sexo y grupos de edad (año 2007).

\begin{tabular}{|c|c|c|c|c|c|c|c|c|c|c|c|c|c|c|c|}
\hline & \multicolumn{5}{|c|}{ Hombres } & \multicolumn{5}{|c|}{ Mujeres } & \multicolumn{5}{|c|}{ Total } \\
\hline 25-34 años & 13.552 & 55 & 405,84 & 305,88 & 527,94 & 15.039 & 122 & 811,22 & 674,11 & 967,84 & 28.591 & 177 & 619,08 & 531,45 & 716,95 \\
\hline 35-44 años & 14.569 & 29 & 199,05 & 133,35 & 285,75 & 16.514 & 132 & 799,32 & 669,20 & 947,19 & 31.083 & 161 & 517,97 & 441,21 & 604,18 \\
\hline $55-64$ años & 11.179 & 8 & 71,56 & 30,90 & 140,96 & 12.838 & 59 & 459,57 & 350,03 & 592,42 & 24.017 & 67 & 278,97 & 216,26 & 354,15 \\
\hline$>64$ años & 16.679 & 12 & 71,95 & 37,18 & 125,64 & 21.739 & 30 & 138,00 & 93,13 & 196,95 & 38.418 & 42 & 109,32 & 78,80 & 147,75 \\
\hline Total & 76.231 & 157 & 205,95 & 175,02 & 240,76 & 88.964 & 482 & 541,79 & 494,61 & 592,24 & 165.195 & 639 & 386,82 & 357,45 & 417,94 \\
\hline
\end{tabular}

Tabla V. Tasa de incidencia (I) × 100.000 habitantes por sexo y grupos de edad (año 2008).



enero de 2004 hasta el 31 de diciembre de 2008; es decir, cinco años completos. De esta manera, la muestra quedó compuesta por 113.096 personas en 2004; 153.105 personas en 2005; 159.233 personas en 2006 ; 165.195 personas en 2007 y 170.362 personas en 2008.

La información que se recogió fue la siguiente: sexo y edad de los pacientes, diagnóstico según la Clasificación Internacional de Enfermedades (CIE-9), especialidades farmacéuticas prescritas, número de recetas y año de prescripción. El código del CIE-9 incluido en el estudio fue el 346.

El cálculo de la incidencia en los cinco años de estudio se realizó utilizando la fórmula habitual (número de casos/población estudiada) con expresión del intervalo de confianza del 95\% (IC 95\%), estrati- ficando la muestra por sexo y grupos de edad (1524 años, 25-34 años, 35-44 años, 45-54 años, 55-64 años y mayores de 64 años). Las posibles diferencias por sexo se analizaron con el test de homogeneidad de porcentajes para una $p<0,05$. Por otro lado, se usaron modelos de regresión de Poisson en todos los grupos de edad estudiados para valorar la tendencia en la incidencia de migraña.

\section{Resultados}

La incidencia de migraña por sexo y grupos de edad se muestra en las tablas I a X. En ellas se indica la tasa de incidencia puntual así como el IC 95\% (por 100.000 habitantes). Las tasas de incidencia varían 
Tabla VI. Comparación de las tasas de incidencia (I) por sexo (año 2004).

\begin{tabular}{|c|c|c|c|c|c|c|c|}
\hline & \multicolumn{3}{|c|}{ Hombres } & \multicolumn{3}{|c|}{ Mujeres } & \multirow{2}{*}{$p$} \\
\hline & $n$ & Casos & $\begin{array}{c}\mathrm{I} \times 100.000 \\
\text { habitantes }\end{array}$ & $n$ & Casos & $\begin{array}{c}\text { I } \times 100.000 \\
\text { habitantes }\end{array}$ & \\
\hline 15-24 años & 4.771 & 11 & 230,56 & 4.877 & 13 & 266,56 & \\
\hline 25-34 años & 8.178 & 22 & 269,01 & 9.688 & 74 & 763,83 & $<0,001$ \\
\hline 35-44 años & 8.948 & 27 & 301,74 & 11.240 & 101 & 898,58 & $<0,001$ \\
\hline 45-54 años & 7.688 & 27 & 351,20 & 10.074 & 85 & 843,76 & $<0,001$ \\
\hline 55-64 años & 7.826 & 11 & 140,56 & 9.526 & 63 & 661,35 & $<0,001$ \\
\hline > 64 años & 12.857 & 12 & 93,33 & 17.423 & 39 & 223,84 & $<0,001$ \\
\hline Total & 50.268 & 110 & 218,83 & 62.828 & 375 & 596,87 & $<0,001$ \\
\hline
\end{tabular}

Tabla VII. Comparación de las tasas de incidencia (I) por sexo (año 2005).

\begin{tabular}{|c|c|c|c|c|c|c|c|}
\hline & \multicolumn{3}{|c|}{ Hombres } & \multicolumn{3}{|c|}{ Mujeres } & \multirow{2}{*}{$p$} \\
\hline & $n$ & Casos & $\begin{array}{c}\mathrm{I} \times 100.000 \\
\text { habitantes }\end{array}$ & $n$ & Casos & $\begin{array}{c}\mathrm{I} \times 100.000 \\
\text { habitantes }\end{array}$ & \\
\hline 15-24 años & 7.169 & 19 & 265,03 & 7.228 & 30 & 415,05 & \\
\hline 25-34 años & 12.184 & 41 & 336,51 & 13.649 & 102 & 747,31 & $<0,001$ \\
\hline 35-44 años & 13.330 & 39 & 292,57 & 15.452 & 123 & 796,01 & $<0,001$ \\
\hline 45-54 años & 11.257 & 23 & 204,32 & 13.401 & 98 & 731,29 & $<0,001$ \\
\hline 55-64 años & 10.433 & 21 & 201,28 & 12.088 & 73 & 603,91 & $<0,001$ \\
\hline > 64 años & 15.977 & 11 & 68,85 & 20.937 & 49 & 234,04 & $<0,05$ \\
\hline Total & 70.350 & 154 & 218,91 & 82.755 & 475 & 573,98 & $<0,001$ \\
\hline
\end{tabular}

Tabla VIII. Comparación de las tasas de incidencia (I) por sexo (año 2006).

\begin{tabular}{|c|c|c|c|c|c|c|c|}
\hline & \multicolumn{3}{|c|}{ Hombres } & \multicolumn{3}{|c|}{ Mujeres } & \multirow{2}{*}{$p$} \\
\hline & $n$ & Casos & $\begin{array}{c}\text { I } \times 100.000 \\
\text { habitantes }\end{array}$ & $n$ & Casos & $\begin{array}{c}\text { I } \times 100.000 \\
\text { habitantes }\end{array}$ & \\
\hline 15-24 años & 7.929 & 25 & 315,30 & 8.157 & 53 & 649,75 & $<0,01$ \\
\hline 25-34 años & 12.779 & 41 & 320,84 & 14.404 & 126 & 874,76 & $<0,001$ \\
\hline 35-44 años & 13.890 & 32 & 230,38 & 16.060 & 125 & 778,33 & $<0,001$ \\
\hline 45-54 años & 11.643 & 21 & 180,37 & 14.030 & 109 & 776,91 & $<0,001$ \\
\hline 55-64 años & 10.848 & 19 & 175,15 & 12.458 & 90 & 722,43 & $<0,001$ \\
\hline$>64$ años & 15.915 & 7 & 43,98 & 21.120 & 34 & 160,99 & $<0,001$ \\
\hline Total & 73.004 & 145 & 198,62 & 86.229 & 537 & 622,76 & $<0,001$ \\
\hline
\end{tabular}

\section{Discusión}

Aunque la migraña es un trastorno común, permanece con frecuencia infradiagnosticada e infratratada [11]. De hecho, se ha propuesto que la incidencia de migraña podría ser más alta de lo que se ha manifestado hasta ahora [12]. Así, parece que alrededor de la mitad de los migrañosos podrían estar infradiagnosticados. En concordancia con esto, un estudio reciente llevado a cabo en Francia mostró que el diagnóstico médico de migraña y su seguimiento podrían ser incompletos [13].

La migraña afecta a alrededor del $12 \%$ de la población en Estados Unidos, y en consecuencia implica un alto coste económico [14-17]. La incidencia de migraña es mayor durante el pico de los años productivos (20-55 años), y más frecuente en muje- 
res, aunque la ratio por sexo varía con la edad [18]. Por ello, los programas de control de la migraña basados en cuestionarios de cribado e iniciativas educacionales pueden disminuir el coste para el empleador derivado de la discapacidad asociada al dolor de cabeza [19].

En el caso de España, la migraña fue, junto con la epilepsia y la neuropatía periférica, una de las enfermedades neurológicas más frecuentes en un estudio con 961 sujetos en un área urbana de Madrid [7]. Igualmente, la prevalencia de migraña se estimó en un $16,6 \%$, con un predominio de mujeres en todos los tipos de migraña, en un estudio puerta a puerta ( $n=790$ sujetos) en la región del Comtat (Alicante, España), usando un autocuestionario con entrevista médica posterior [20]. Sin embargo, en el caso de la migraña crónica su prevalencia global se ha estimado en un rango del 0,9-5,1\% [21].

La incidencia de la migraña en el Reino Unido se ha estimado recientemente en 0,369 (casos por 100 personas/año) [22]. En el caso de EE. UU., la incidencia media anual fue 0,343 por 1.000 personas/ año $(0,4816$ en mujeres y 0,1944 en hombres) en Minnesota [23]. Nuestros resultados muestran una incidencia de migraña en Álava similar a la comunicada en otros países, sin cambios significativos en el período 2004-2008 ( $p=0,189)$.

Sin embargo, hemos de remarcar que nuestros resultados corresponden a la incidencia diagnóstica de migraña y no a la incidencia real, posiblemente mayor debido al infradiagnóstico. Así, en un estudio realizado en Dinamarca [24], se obtuvieron cifras de incidencia de 8,1 por 1.000 personas/año, si bien la muestra estaba compuesta por sujetos de 25 a 64 años.

Dado que la aparición de la migraña se ha relacionado con las condiciones socioeconómicas [25], estos resultados sugieren una buena calidad de vida de la población del País Vasco en los últimos años, lo que indica una confluencia con otras regiones europeas. También como en nuestro trabajo, la incidencia de migraña en el Reino Unido es 2,5 veces mayor en las mujeres con respecto a los hombres [26].

La incidencia de migraña en EE. UU. alcanza un pico a los 20-24 años en las mujeres y a los 15-19 años en los hombres [23]. En nuestro trabajo la incidencia de la migraña en los hombres fue máxima a la edad de 45-54 años en 2004 y en las franjas de 25-34 años en 2005, 2006, 2007 y 2008. En las mujeres, la edad de máxima aparición fue de 35-44 años en 2004 y 2005 y de 25-34 años en 2006, 2007 y 2008. Considerando a los hombres y las mujeres juntos, la edad de máxima aparición de la migraña fue de 35-44 años en 2004 y 2005 y de 25-34 años
Tabla IX. Comparación de las tasas de incidencia (I) por sexo (año 2007).

\begin{tabular}{|c|c|c|c|c|c|c|c|}
\hline & \multicolumn{3}{|c|}{ Hombres } & \multicolumn{3}{|c|}{ Mujeres } & \multirow[b]{2}{*}{$p$} \\
\hline & $n$ & Casos & $\begin{array}{l}\text { I } \times 100.000 \\
\text { habitantes }\end{array}$ & $n$ & Casos & $\begin{array}{c}\mathrm{I} \times 100.000 \\
\text { habitantes }\end{array}$ & \\
\hline 15-24 años & 8.239 & 19 & 230,61 & 8.381 & 44 & 525,00 & $<0,01$ \\
\hline 25-34 años & 13.552 & 55 & 405,84 & 15.039 & 122 & 811,22 & $<0,001$ \\
\hline 35-44 años & 14.569 & 29 & 199,05 & 16.514 & 132 & 799,32 & $<0,001$ \\
\hline 45-54 años & 12.013 & 34 & 283,03 & 14.453 & 95 & 657,30 & $<0,001$ \\
\hline 55-64 años & 11.179 & 8 & 71,56 & 12.838 & 59 & 459,57 & $<0,001$ \\
\hline > 64 años & 16.679 & 12 & 71,95 & 21.739 & 30 & 138,00 & \\
\hline Total & 76.231 & 157 & 205,95 & 88.964 & 482 & 541,79 & $<0,001$ \\
\hline
\end{tabular}

Tabla X. Comparación de las tasas de incidencia (I) por sexo (año 2008).

\begin{tabular}{|c|c|c|c|c|c|c|c|}
\hline & \multicolumn{3}{|c|}{ Hombres } & \multicolumn{3}{|c|}{ Mujeres } & \multirow[b]{2}{*}{$p$} \\
\hline & $n$ & Casos & $\begin{array}{c}\text { I } \times 100.000 \\
\text { habitantes }\end{array}$ & $n$ & Casos & $\begin{array}{c}\text { I } \times 100.000 \\
\text { habitantes }\end{array}$ & \\
\hline 15-24 años & 8.244 & 20 & 242,60 & 8.775 & 61 & 695,16 & $<0,001$ \\
\hline 25-34 años & 14.042 & 49 & 348,95 & 15.839 & 141 & 890,21 & $<0,001$ \\
\hline 35-44 años & 15.063 & 51 & 338,58 & 17.044 & 123 & 721,66 & $<0,001$ \\
\hline 45-54 años & 12.547 & 34 & 270,98 & 14.969 & 107 & 714,81 & $<0,001$ \\
\hline 55-64 años & 11.798 & 14 & 118,66 & 13.271 & 54 & 406,90 & $<0,001$ \\
\hline > 64 años & 16.891 & 5 & 29,60 & 21.879 & 24 & 109,69 & $<0,001$ \\
\hline Total & 78.585 & 173 & 220,14 & 91.777 & 510 & 555,70 & $<0,001$ \\
\hline
\end{tabular}

en 2006, 2007 y 2008. Así, aunque los nuevos casos de migraña tendieron a aparecer preferentemente en las edades más jóvenes en el período 2004-2008, estos resultados sugieren que muchos migrañosos podrían estar infradiagnosticados.

$\mathrm{Al}$ igual que en EE. UU., la edad de máxima aparición de la migraña en las mujeres mostró una tendencia a disminuir en Álava, lo que sugiere una posible relación con una aparición más precoz de la menarquia [27]. Así, la edad media de aparición de la menarquia en Cataluña (España) se estimó en 12,31 años, similar a otros países mediterráneos y menor que en otras zonas de España y los países del norte de Europa [28]. Concretamente, la edad de la menarquia en la costa de Vizcaya (País Vasco) es $12,75 \pm 0,04$ años [29]. 
Figura 1. Tendencia de las tasas de incidencia a lo largo de los años estudiados.

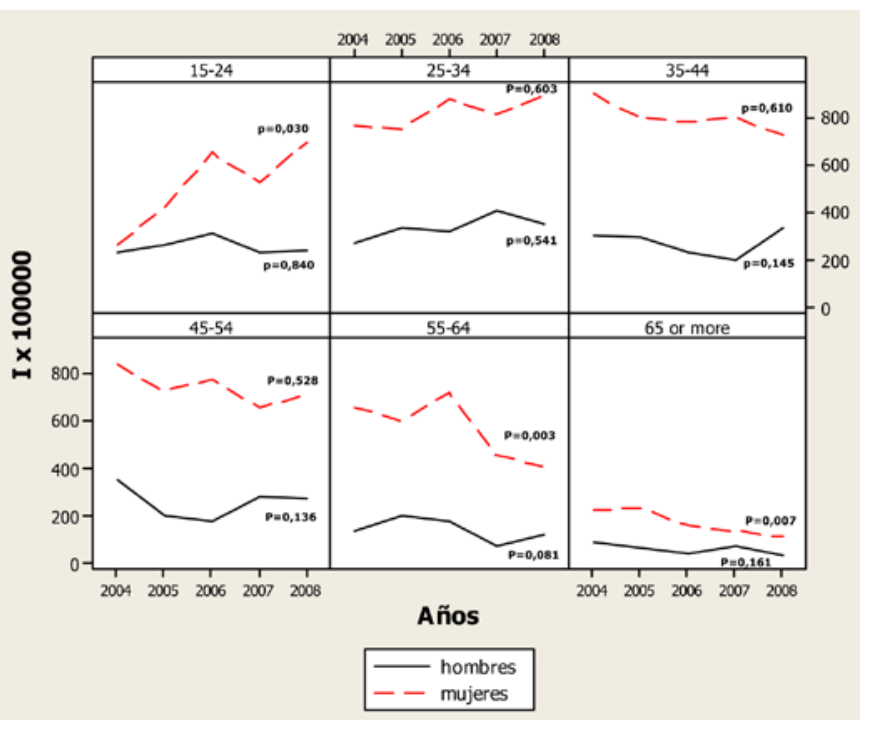

Figura 2. Tendencia de las tasas de incidencia a lo largo de los años estudiados en la población total.

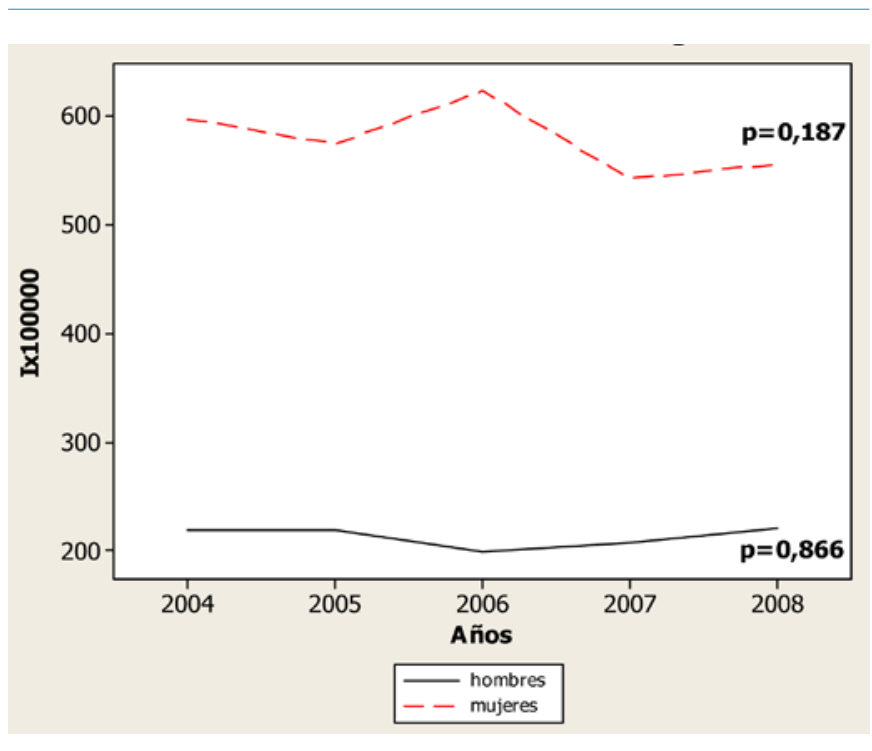

La migraña en España es más frecuente en mujeres entre 31 y 50 años, y se asocia a ingresos bajos, mala calidad del sueño, mal estado de salud, depresión y otras condiciones de comorbilidad, principalmente dolor crónico en el cuello y asma [9]. Teniendo en cuenta que la migraña (y sobre todo la que tiene aura) es un factor de riesgo cerebrovascular, se debería prestar una atención especial a las mujeres jóvenes en el diseño de programas de prevención y control clínico de la migraña.

En conclusión, nuestros datos muestran una tendencia al aumento de la incidencia de migraña en mujeres de edades jóvenes y una disminución de ésta en personas de edad avanzada en Álava.

\section{Bibliografía}

1. Cutrer FM. Pathophysiology of migraine. Semin Neurol 2010; 30: $120-30$.

2. Supiot F. Migraine in 2009: from attack to treatment. Rev Med Brux 2009; 30: 399-403.

3. Del Sette M. Migraine and cerebrovascular risk. Neurol Sci 2010; 31 (Suppl 1): S127-8.

4. Gudmundsson LS, Scher AI, Aspelund T, Eliasson JH, Johannsson M, Thorgeirsson G, et al. Migraine with aura and risk of cardiovascular and all cause mortality in men and women: prospective cohort study. BMJ 2010; 341: c3966.

5. Bigal ME, Lipton RB, Stewart WF. The epidemiology and impact of migraine. Curr Neurol Neurosci Rep 2004; 4: 98-104.

6. Yu J, Goodman MJ, Oderda GM. Economic evaluation of pharmacotherapy of migraine pain: a review of the literature. J Pain Palliat Care Pharmacother 2009; 23: 396-408.
7. Cruz-Gutiérrez del Olmo M, Schoenberg BS, PorteraSánchez A. Prevalence of neurological diseases in Madrid, Spain. Neuroepidemiology 1989; 8: 43-7.

8. Montiel I, Muñiz R, Asensio M, Ruiz C, González MJ, Díaz-Marín C, et al. Prevalencia de migraña en una población homogénea utilizando el autocuestionario 'Alcoi-1995' Rev Neurol 1997; 25: 1177-80.

9. Fernández de las Peñas C, Hernández-Barrera V, CarrascoGarrido P, Alonso-Blanco C, Palacios-Ceña D, JiménezSánchez S, et al. Population-based study of migraine in Spanish adults: relation to socio-demographic factors, lifestyle and co-morbidity with other conditions. J Headache Pain 2010; 11: 97-104.

10. Matías-Guiu J, Porta-Etessam J, Mateos V, Díaz-Insa S, López-Gil A, Fernández C. One-year prevalence of migraine in Spain: a nationwide population-based survey. Cephalalgia 2011; 31: 463-70.

11. Lipton RB, Stewart WF, Reed M, Diamond S. Migraine's impact today. Burden of illness, patterns of care. Postgrad Med 2001; 109: 38-40, 43-5.

12. Kobak KA, Katzelnick DJ, Sands G, King M, Greist JJ, Dominski MJ. Prevalence and burden of illness of migraine in managed care patients. Manag Care Pharm 2005; 11: 124-36.

13. Lucas C, Géraud G, Valade D, Chautard MH, Lantéri-Minet M. Recognition and therapeutic management of migraine in 2004, in France: results of FRAMIG 3, a French nationwide population-based survey. Headache 2006; 46: 715-25.

14. Mathew NT. Pathophysiology, epidemiology, and impact of migraine. Clin Cornerstone 2001; 4: 1-17.

15. $\mathrm{Hu}$ XH, Markson LE, Lipton RB, Stewart WF, Berger ML. Burden of migraine in the United States: disability and economic costs. Arch Intern Med 1999; 159: 813-8.

16. Stewart WF, Lipton RB. The economic and social impact of migraine. Eur Neurol 1994; 34 (Suppl 2): S12-7.

17. Láinez JM. Impacto socioeconómico de la migraña en el medio laboral. Medicina del Trabajo 1994; 3: 113-6.

18. Lipton RB, Stewart WF, Scher AI. Epidemiology and economic impact of migraine. Curr Med Res Opin 2001; 17 (Suppl 1): S4-12. 
19. Page MJ, Paramore LC, Doshi D, Rupnow MF. Evaluation of resource utilization and cost burden before and after an employer-based migraine education program. J Occup Environ Med 2009; 51: 213-20.

20. Sánchez-Pérez R, Asensio M, Melchor A, Montiel I, Falip R Moltó JM, et al. Estudio descriptivo de migraña en una población rural del Àrea del Comtat. Rev Neurol 1999; 28: 373-6.

21. Natoli J, Manack A, Dean B, Butler Q, Turkel C, Stovner L, et al. Global prevalence of chronic migraine: a systematic review. Cephalalgia 2010; 30: 599-609.

22. Becker C, Brobert GP, Almqvist PM, Johansson S, Jick SS, Meier CR. Migraine incidence, comorbidity and health resource utilization in the UK. Cephalalgia 2008; 28: 57-64.

23. Rozen TD, Swanson JW, Stang PE, McDonnell SK, Rocca WA Incidence of medically recognized migraine: a 1989-1990 study in Olmsted County, Minnesota. Headache 2000; 40: 216-23.

24. Lyngberg AC, Rasmussen BK, Jorgensen T, Jensen R. Incidence of primary headache: a Danish epidemiologic follow-up study. Am J Epidemiol 2005; 161: 1066-73.
25. Vidal-Jordana A, Barroeta-Espar I, Sáinz-Pelayo MP, Sala I, Roig C. Cefalea e inmigración. Estudio en la consulta externa del Hospital de la Santa Creu i Sant Pau de Barcelona. Rev Neurol 2011; 53: 275-80.

26. Stewart WF, Wood C, Reed ML, Roy J, Lipton RB; AMPP Advisory Group. Cumulative lifetime migraine incidence in women and men. Cephalalgia 2008; 28: 1170-8.

27. Cabanes A, Ascunce N, Vidal E, Ederra M, Barcos A, Erdozain N, et al. Decline in age at menarche among Spanish women born from 1925 to 1962. BMC Public Health 2009; 9: 449 .

28. De la Puente ML, Canela J, Álvarez J, Salleras L, VicensCalvet E. Cross-sectional growth study of the child and adolescent population of Catalonia (Spain). Ann Hum Biol 1997; 24: 435-52.

29. Rebato E, Rosique J, González-Apraiz A. Age at menarche from a Biscayan coastal population (Basque Country). Ann Hum Biol 1993; 20: 191-3.

\section{Evolution of the incidence of migraine in Álava over the period 2004-2008}

Introduction. Few studies have been conducted on the incidence of migraine in Spain, and those that have been carried out have some shortcomings, such as the lack of a clear population base.

Aims. To examine the evolution of the incidence of migraine over the period 2004-2008 in the province of Álava (Basque Country, Spain).

Patients and methods. Data concerned persons aged 15 or over who were registered in the Basque Health Service/Osakidetza database as new cases diagnosed with migraine. The tendency of the incidence of migraine was evaluated by means of linear regression.

Results. The incidence of migraine was significantly higher in females than in males, at any age. No significant changes in the incidence of migraine were observed over the period 2004-2008 in the population of Ávala as a whole $(p=0.189)$. Nevertheless, there have been both a decrease in the incidence of migraine in females over the age of $64(p=0.014)$ and an increase in those aged $15-24$ years $(p=0.052)$ and 35-44 years $(p=0.057)$. The new cases of migraine that are diagnosed tended to appear at younger ages over this period.

Conclusions. These results suggest an absence of changes in the tendency in the incidence of migraine throughout the period 2004-2008, except for a decrease in the case of females over 64 years of age and an increase in young females. This evolution is similar to that of other regions in Europe.

Key words. Incidence. Migraine. 